\title{
Dimensiones de evaluación de la calidad universitaria en el Espacio Europeo de Educación Superior
}

\author{
Ignacio González López
}

Facultad de Ciencias de la Educación, Universidad de Córdoba

\section{España}

ed1goloi@uco.es 


\section{Resumen}

Introducción: el presente trabajo trata de establecer las dimensiones básicas sobre las cuáles construir un sistema de indicadores de evaluación de la calidad universitaria desde la perspectiva del alumnado, en el marco ofrecido por el Espacio Europeo de Educación Superior.

Método: la población de este estudio quedó definida por el conjunto de alumnos y alumnas matriculadas en las Universidades de Salamanca (USAL) y Córdoba (UCO) en el curso académico 2004/2005, que asciende a un total de 45751 estudiantes. Se trata de dos instituciones localizadas en diferentes puntos geográficos del territorio español y con unas señas de identidad propias. La muestra obtenida estuvo compuesta por un total de 1167 sujetos estratificados en función de su rama de especialización: ciencias de la salud, humanidades, jurídicosociales y técnicas. Para la recogida de información recurrimos al diseño de un cuestionario formado por un protocolo de ítems de valoración escalar. La aplicación de un análisis factorial nos permitió establecer las dimensiones básicas a partir de las cuáles establecer los diferentes indicadores de evaluación.

Resultados: los resultados obtenidos han aportado un total de 14 factores entre los que destacan, por su potencialidad y utilidad, la satisfacción del alumnado, las competencias académicas y profesionales, la evaluación del rendimiento académico, la enseñanza virtual y la acción tutorial.

Discusión: las diferentes pruebas llevadas a cabo han evidenciado que la calidad se define, en primer lugar, por la satisfacción del alumnado, resultados que vienen a refrendar la lógica de los modelos de evaluación institucional implementados en España.

Palabras Clave: Espacio Europeo de Educación Superior, indicadores de calidad, evaluación educativa, calidad educativa 


\section{Introducción}

La resolución de 23 de marzo de 2005 de la Secretaría de Estados de Universidades e Investigación (BOE $\mathrm{n}^{\circ} 84$, de 8 de abril de 2005) decidió apostar por una propuesta destinada a mejorar la enseñaza superior y la actividad del profesorado universitario (referencia EA2005-0152), cuyo objetivo ha sido la construcción de un sistema de indicadores de evaluación de la calidad universitaria desde la perspectiva del alumnado, en el marco ofrecido por el Espacio Europeo de Educación Superior.

Reconociendo que la verdadera calidad de la universidad, tal y como señala Rodríguez (1991: 41), radica en su "capacidad para lograr el mayor desarrollo posible de sus miembros" y considerando la "evaluación como un proceso de reflexión valorativa y sistémica, que contribuye decididamente a la mejora de la calidad de la educación" (Ruiz, 1996: 15), es preciso situarse en la perspectiva de la evaluación institucional, que trata de dar una visión global de los aspectos constitutivos de la universidad y advertir la manera en que ésta cumple con sus ojetivos. El acceso a esta información, en organizaciones de gran tamaño, convierte a los indicadores en el elemento fundamental para emplear en un proceso de mejora (Apodaca y Grao, 1996).

Es por ello que, gracias a la información aportada por estudiantes de dos instituciones universitarias de carácter histórico y formativo diferente, en este artículo se presentan los resultados referentes a la dimensiones que han servido de base para la construcción de un sistema de indicadores de evaluación.

\section{Hacia una nueva concepción de calidad educativa}

La puesta en marcha de un Espacio Europeo de Educación Superior antes del año 2010 es uno de los objetivos prioritarios de la Unión Europea, tanto para los países integrantes como para aquellos que están a punto de ingresar. Su propósito es generar un sistema educativo de calidad, a través de una formación armónica y adecuada a los requisititos del sistema productivo europeo. Se trata de un proceso imparable de armonización de los sistemas de enseñanza superior que requiere de una labor conjunta entre universidades y centros de enseñanza superior, instituciones y países. 
Conscientes del papel que desempeña la educación en el desarrollo de los ciudadanos y las sociedades modernas, el 19 de junio de 1999, treinta y un países europeos firmaron una declaración conjunta (Declaración de Bolonia, 1999) que supone el punto de partida del proceso de convergencia hacia un Espacio Europeo de Educación Superior, bajo los principios de calidad, movilidad, diversidad y competitividad. El objetivo del desarrollo de este espacio supone dotar a Europa de un sistema educativo homogéneo, compatible y flexible, que permita a los estudiantes y titulados universitarios europeos una mayor movilidad, dotando de vitalidad y eficacia los diversos canales por los que el alumnado se forma. Es el estudiante el centro de este sistema, su principal referente, el actor por excelencia, por lo que se ha de valorar su formación más allá, aún sabiendo la importancia que hasta este momento se dota a la acumulación de conocimientos. Se trata de dotarle de las competencias necesarias para desenvolverse profesional y personalmente potenciando su autonomía, el pragmatismo, el trabajo en equipo y la formación permanete recurriendo, entre otros, al empleo de las tecnologías de la información y de la comunicación y la acción orientadora del docente. Es por ello que se plantea la necesidad de establecer los principios básicos que guiarán esta formación en el ámbito de competencias, metodologías, herramientas y sistemas de evaluación. Se trata de dar una respuesta común y efectiva a las demandas planteadas y al nuevo contexto surgido de una política económica, cultural y social internacional.

En la Convención de Instituciones Europeas de Educación Superior (Convención de Salamanca, 2001), celebrada los días 29 y 30 de marzo de 2001, donde se reitera el apoyo a los principios de la Declaración de Bolonia, la calidad fue considerada como la base fundamental, la condición imprescindible para la confianza, pertinencia, movilidad, compatibilidad y atractivo en el Espacio Europeo de Educación Superior. Esta garantía supone un equilibrio entre la innovación y la tradición, entre la excelencia académica y la pertinencia social y económica, significa disponer de unos currícula coherentes a las demandas del mercado de trabajo y del sistema social y precisa dotar al estudiante de libertad de decisión.

Dos años después de la firma de la Declaración de Bolonia, tuvo lugar en Praga, el 19 de mayo de 2001, una reunión de seguimiento donde los ministros allí reunidos adoptaron un Comunicado (Comunicado de Praga, 2001) que supone la ratificación de los progresos realizados hasta la fecha y donde se concretan las medidas que se deben llevar a cabo para conseguir la puesta en marcha del Espacio Europeo de Educación Superior en 2010. Entre las conclusiones más relevantes de esta reunión destaca la promoción de la cooperación europea en 
el control y aseguramiento de la calidad, reconociendo el papel fundamental de estos sistemas a la hora de garantizar unos niveles óptimos de excelencia.

Un nuevo avance en este proceso lo consigue la Conferencia de Berlín (2003), celebrada el 19 de septiembre de 2003, una reunión que sirvió para analizar los logros alcanzados y establecer las prioridades y los nuevos objetivos para las próximas etapas de construcción del Espacio Europeo de Educación Superior. Uno de los parámetros a continuar es el desarrollo de sistemas que garanticen la calidad de la educación superior como una de las piezas fundamentales del establecimiento de este espacio común. Bajo el principio de autonomía institucional, se considera necesario establecer criterios y metodologías comunes que han de incorporar los sistemas nacionales de garantía de calidad de los diferentes países.

Antes de la siguiente reunión en Londres en 2007, los Ministros de Educación europeos tuvieron la oportunidad de reunirse en Bergen los días 19 y 20 de mayo, donde se dio un empuje a las medidas adoptadas en Berlín y procurando un alto grado de cooperación y de formación de redes (Comunicado de Bergen, 2005). Sin embargo, se dieron cuenta de que hay que progresar bastante aún, particularmente en lo que se refiere a la participación de los estudiantes y la cooperación internacional. Aún más, instan a las instituciones de Educación Superior a continuar los esfuerzos para incrementar la calidad de sus actividades, a través de la introducción sistemática de mecanismos internos y su correlación directa con la garantía de calidad externa.

\section{¿Cómo garantizar la calidad?}

La mejora de la calidad del sistema universitario es una pieza clave sobre la que pivota el compromiso de crear un área europea de Educación Superior, un lugar de referencia en el ámbito de la formación universitaria que precisa de mecanismos y procesos de evaluación, certificación y acreditación.

Diferentes estudios realizados por Gento (1996), Álvarez (1998), Martín (2001) y González (2004), sobre los aportes a la calidad en educación superior desde diferentes modelos y metodologías de evaluación, concluyen que la garantía de este principio en la construcción del espacio educativo común europeo debe de respetar una serie de requisitos como los que aquí se exponen: 
- El estudiante es lo fundamental, por lo tanto, satisfacer al estudiante es lo más importante. Es preciso que las necesidades y expectativas del alumnado, como principal beneficiario de la formación universitaria, sean cubiertas. Por otra parte, el personal (docente, de investigación, de administración y de servicios) ha de sentirse satisfecho con su tarea diaria y ser considerado un motor de avance dentro de la organización.

- La gestión de la calidad se fundamenta en el desarrollo de un proceso continuo y permanente. Los programas de mejora continua son preferibles a las soluciones rápidas.

- La toma de decisiones se produce como consecuencia de datos y evidencias, no sobre suposiciones y opiniones. Se trata de procesos de actuación sistemáticos y estructurados, basados en información de muy diferente naturaleza, obtenidas a través del empleo de instrumentos de recogida de datos como las entrevistas, los cuestionarios, estadísticas oficiales, grupos de discusión, etc.

- Se fundamenta en proponer soluciones y no sólo en señalar problemas o deficiencias. Con la evaluación tratamos de detectar aquellos puntos fuertes y débiles de la institución y, en consecuencia, generar propuestas que den una solución efectiva a los problemas planteados y prevengan dificultades posteriores.

- La calidad depende básicamente de las personas, por tanto, es fundamental la colaboración, el trabajo en equipo, la participación, el compromiso, la implicación voluntaria, la formación del personal y el desarrollo y crecimiento personal de cada individuo como clave de crecimiento y enriquecimiento de la organización.

- La calidad implica a toda la organización directa o indirectamente, incluido el alumnado como indiscutible referente.

Ello implica que la diversidad de procedimientos que garanticen la calidad educativa en Europa suponga un reto en la homogeneización de los procedimientos necesarios para su producción y mantenimiento. Existen modelos centrados en la evaluación de programas y 
modelos centrados en la calidad total, agencias estatales y agencias autónomas, lo que ha conllevado a la creación de la Asociación de Universidades Europeas en Salamanca, en abril de 2001, cuya misión fundamental es contribuir al desarrollo del espacio europeo para la educación superior y la investigación, ayudando a fortalecer la calidad. Este organismo proporciona a sus miembros el Programa de Revisión Institucional (que evalúa la gestión estratégica y la capacidad de cambio de las instituciones) y el Proyecto de Calidad de la Cultura (destinado a desarrollar un cultura interna de calidad en las instituciones).

Del mismo modo, la falta de consenso y la heterogeneidad de una serie de acciones destinadas al mismo fin ha llevado a la Comisión Europea a promover el Proyecto Tuning (González y Wagenaar, 2003), que pretende crear un índice de referencia para la elaboración y evaluación de los planes de estudio. Se trata de armonizar un lenguaje común que permita la flexibilidad y la autonomía para el cumplimiento de los objetivos de unidad, diversidad y calidad propuestos.

¿Qué es preciso evaluar?

La demanda de formación universitaria va a seguir creciendo, aunque no tan rápido como hasta ahora. Además, la perspectiva marcada por el proceso de convergencia europea va a cambiar de forma bastante profunda la estructura de la demanda, nuevos colectivos accederán a la Universidad y se demandarán nuevos servicios universitarios. Al mismo tiempo, se exigirá de ella un nivel aceptable de calidad, tanto en la investigación como en la docencia, pero, sobre todo, cada vez va a tener mayor importancia la exigencia de calidad en los servicios universitarios. Por último, se requerirá que sea una Universidad más flexible, tanto en sus estructuras organizativas como en su oferta de enseñanzas y de servicios a la colectividad.

Como se puede apreciar, el problema de la calidad de la educación no es simple. Este nuevo entorno potencia la formación de profesionales que posean conocimientos científicos de su actividad así como una serie de competencias culturales, emocionales y sociales que les formen como personas, tal y como han reflejado anteriormente los estudios de Hernández (1997), Chadwick y Thorne de Trelles (1998) o Álvarez (2000). De ellos podemos destacar competencias académico-profesionales centradas principalmente en la resolución de conflictos, la transferencia de los conocimientos adquiridos a situaciones de la vida real y la construcción de un espíritu crítico. En definitiva, la institución universitaria ha de atender a las 
cuatro dimensiones planteadas por Rodríguez (1998) a la hora de construir la personalidad humana: cognitiva, volitiva, tecnológica y social. Por lo tanto, esta complejidad se asocia a una multiplicidad de factores sobre los cuáles se debería actuar para obtener como resultado una educación más extensa, tal y como anuncian modelos tales como el Premio Deming, el Premio Baldridge, la Organización Internacional para la Estandarización y el Modelo Europeo de Gestión de Calidad: fines y metas de la institución, roles y funciones de sus miembros, gobierno y representación, formación académica, metodología docente, instalaciones y recursos y satisfacción. Aunque no está claro cuál es el peso relativo de cada uno de esos factores, sí se sabe que sin mejores profesores, sin unos currícula renovados y sin alumnos con capacidad y voluntad de estudiar, no hay posibilidad alguna de tener buenos resultados.

\section{Método}

Cuando se habla de dimensiones de evaluación de la calidad en la universidad una de las más relevantes es la referida al alumnado, en lo relativo básicamente a matriculación, absentismo, calificaciones, etc. Sin embargo, es curioso observar la falta de estudios evaluativos acerca de la opinión de este grupo poblacional sobre los que para él supone una universidad de calidad y cuáles serían los elementos que la reflejan. Es por ello que en este trabajo necesitamos recoger la opinión de los estudiantes sobre lo que para ellos supone una Universidad de calidad en la perspectiva planteada por la construcción del Espacio Europeo de Educación Superior y cuáles son las dimensiones más importantes sobre las que centrar esta calidad, en qué grado están implicados o quieren estar implicados en la Institución, qué grado de información tienen sobre ella y cuál es su nivel de satisfacción actual sobre todos los procesos que se desarrollan en la Universidad.

Tras la formulación de estas metas, hemos establecido las variables que dan cuenta de los fenómenos a estudiar. Para su selección hemos considerado aquellos factores que condicionan la calidad de una institución universitaria en el ámbito europeo. Estos son muy variados y de muy diversa índole, por lo que se han empleado dos criterios para su definición. Por un lado, hemos considerado la propia naturaleza de las variables, de ahí que aparezcan: características del alumnado, variables actitudinales, variables relacionadas con la implicación de los alumnos en la comunidad universitaria, variables relacionadas con las actividades de formación realizadas por los estudiantes, variables relacionadas con la inserción laboral, varia- 
bles relacionadas con la satisfacción de los estudiantes y variables relacionadas con la calidad universitaria.

Para recoger la información referente a las variables aquí tratadas recurrimos al diseño de un cuestionario autoaplicado o autoadministrado, formado por un protocolo de ítems de valoración escalar y una serie de preguntas abiertas donde el alumnado tuvo libertad de expresión.

La población de este estudio quedó definida por el conjunto de alumnos y alumnas matriculadas en primeros y últimos cursos de la totalidad de titulaciones existentes en la en las Universidades de Salamanca (USAL) y Córdoba (UCO) en el curso académico 2004/2005, que asciende a un total de 45751 estudiantes (15600 procedentes de la UCO y 30151 procedentes de la USAL). Se trata de dos instituciones localizadas en diferentes puntos geográficos del territorio español y con unas señas de identidad propias. En tanto la UCO es relativamente joven (su historia se remonta a escasos 50 años), la USAL está a punto de cumplir ocho siglos de historia. El carácter formativo de la primera de ellas es eminentemente experimental y técnico, mientras que la USAL apuesta por la formación humanística, jurídica y biomédica principalmente.

Dada la diversidad de titulaciones existentes, se optó por seguir un proceso de muestreo aleatorio estratificado en función de las ramas de especialización: Ciencias de la Salud, Experimentales, Humanidades, Jurídico-Sociales y Técnicas. A continuación, seleccionamos de forma aleatoria 51 grupos de 19 titulaciones del abanico de posibilidades que ofrecían la UCO y la USAL con presencia de los estratos aquí definidos. Para establecer la representatividad de la muestra seleccionada llevamos a cabo una afijación proporcional distribuyendo a los elementos de acuerdo con el peso relativo de la población de cada estrato. A continuación devolvimos la proporcionalidad a cada estrato ponderando en función de la población de la cual fue extraída.

La muestra obtenida estuvo compuesta por un total de 1167 sujetos sobre la base de los criterios de estratificación aquí presentados (ver tablas 1 y 2). Ésta ha quedado condicionada por una serie de factores que se comentan a continuación. En primer lugar, se necesitaron los permisos de las autoridades académicas para ocupar un reducido espacio de tiempo (25 minutos) en las unidades de docencia seleccionadas. En segundo lugar, era preciso contac- 
tar con los profesores de los grupos escogidos y contar con su aprobación para ocupar parte de su horario docente. Por último, la fecha de aplicación del cuestionario (abril-mayo del año 2005) coincidió con el final del segundo cuatrimestre y con el inicio de los exámenes de fin de curso, por lo que el nivel de asistencia del alumnado a las aulas ha sido un factor esencial en la composición final de la muestra.

Tabla 1: Población, muestra idónea y muestra real de la UCO

\begin{tabular}{l|c|c|c|c|c|c}
\multirow{2}{*}{\multicolumn{2}{c}{ Estratos }} & \multicolumn{2}{c|}{ Población } & \multicolumn{2}{c|}{ Muestra idónea } & \multicolumn{2}{c}{ Muestra real } \\
\cline { 2 - 7 } & F & fr (\%) & f & fr (\%) & f & fr (\%) \\
\hline \multicolumn{1}{c|}{ Ciencias Salud } & 2689 & 17.2 & 58 & 17.2 & 0 & 0 \\
\hline Experimentales & 1893 & 12.1 & 41 & 12.1 & 35 & 10.3 \\
\hline Humanidades & 1363 & 8.7 & 29 & 8.7 & 78 & 23.3 \\
\hline Jurídico-Sociales & 5296 & 34 & 115 & 34 & 157 & 46.3 \\
\hline Técnicas & 4359 & 28 & 95 & 28 & 68 & 20.1 \\
\hline Total & 15600 & 100 & 338 & 100 & 338 & 100
\end{tabular}

Tabla 2: Población, muestra idónea y muestra real de la USAL

\begin{tabular}{l|c|c|c|c|c|c}
\multirow{2}{*}{\multicolumn{1}{c|}{ Estratos }} & \multicolumn{2}{c|}{ Población } & \multicolumn{2}{c|}{ Muestra idónea } & \multicolumn{2}{c}{ Muestra real } \\
\cline { 2 - 7 } & F & fr $(\%)$ & f & fr (\%) & f & fr (\%) \\
\hline \multicolumn{1}{c|}{ Ciencias Salud } & 4521 & 15 & 124 & 15 & 193 & 23.3 \\
\hline Experimentales & 3135 & 10.4 & 86 & 10.4 & 13 & 1.5 \\
\hline Humanidades & 5277 & 17.5 & 146 & 17.5 & 228 & 27.5 \\
\hline Jurídico-Sociales & 12476 & 41.4 & 343 & 41.4 & 304 & 36.7 \\
\hline Técnicas & 4742 & 15.7 & 130 & 15.7 & 91 & 11 \\
\hline Total & 30151 & 100 & 829 & 100 & 829 & 100
\end{tabular}

\section{Resultados}

La técnica del análisis factorial es utilizada para reducir un amplio número de fenómenos, conceptos o variables, a un número más reducido de componentes o factores, de tal modo que sean representativos de esos conceptos. Así, lo que se busca es que todas las variables entre las que existe una relación se agrupen o saturen en un mismo factor, utilizándose como método exploratorio. Se supone que todas ellas poseen un atributo común que es etiquetado por el investigador, dando así nombre al factor. 
Esta técnica se puede utilizar para estudiar la estructura de los instrumentos de manera que podamos afirmar que determinados ítems se explican mejor desde una dimensión que desde otra e incluso aconsejar la eliminación de ciertos reactivos dada su escasa consistencia interna con los demás ítems de las diferentes dimensiones identificadas. Esta es la razón por la que García, Gil y Rodríguez (2000: 16-17) afirman que el análisis factorial puede ser empleado como un procedimiento básico para el estudio de la calidez de las inferencias hechas a partir de la aplicación del instrumento de medida y la fiabilidad de dicha medida.

Previa selección de los métodos de extracción (componentes principales: buscando el subespacio de menor dimensión que conserve la mayor cantidad de varianza posible) y rotación (varimax: buscando la independencia entre factores), nos propusimos realizar el análisis factorial con los datos procedentes de los 1167 alumnos encuestados para especificar las dimensiones sobre las cuáles basar las propuestas de elaboración de estándares que, dentro de la perspectiva del Espacio Europeo de Educación Superior, valoren la calidad de las instituciones universitarias. Un valor de Barlett de 28503.75, significativo a un nivel de $0.01(\mathrm{p}=0)$, indica que la matriz de datos es adecuada para aplicar ese técnica. Misma discusión aportan las correlaciones anti-imagen que, en su mayoría, son menores de 0.05. Asimismo, el valor de Kaiser-Meyer-Olkin ha sido de 0.889 , lo que aconseja el empleo del análisis factorial.

El análisis ha de realizarse sobre las variables que hagan referencia a un mismo concepto genérico. Dado que el cuestionario empleado en este estudio contiene preguntas de diferente tipo, el análisis ha sido realizado sobre 105 ítems cerrados de elección (escalas ordinales), tipología de variables idóneas para la realización de esta actividad.

El objetivo principal de esta fase es determinar el número mínimo de factores comunes capaces de reproducir, de un modo satisfactorio, las correlaciones observadas entre las variables.

Realizamos este proceso a partir del método de extracción de componentes principales, cuyo objetivo es maximizar la varianza explicada. El factor que mejor explique la dimensión analizada (el que represente mayor variabilidad) se convertirá en el primer componente principal y así sucesivamente. Su aplicación supone transformar directamente un conjunto de variables correlacionadas en un conjunto de variables no correlacionadas (García, Gil y Rodríguez, 2000: 27). 
El objetivo consiste en maximizar la explicación de esa varianza con el menor número de factores, aspecto que determinará el total de factores a extraer. Partiendo la regla de conservar aquellos componentes cuyos autovalores son mayores que la unidad, obtuvimos un total de 27 factores con una explicación total de la varianza de un $62.869 \%$, lo que dificultaba los trabajos posteriores y donde la técnica no presuponía su efectividad. Según las ideas manifestadas por García, Gil y Rodríguez (2000: 22), el número mínimo de variables que debe configurar un factor debe ser mayor de tres, dado que con un número más reducido es claro que matemáticamente encontraremos un único factor que aglutine la información de las correlaciones entre variables. Hasta llegar a un buen modelo factorial necesitaremos definir gradualmente la muestra de variables que mejor representa el dominio de un estudio eliminando los factores de menor importancia (los que menor varianza expliquen o los de contenido menos general). Hasta el factor número catorce son al menos dos las variables que componen cada factor, siendo su correlación con el mismo alta. A partir del factor 15, cada uno de ellos asume menos de un $2 \%$ de la varianza total explicada. Por estas razones y dado que los catorce primeros factores explican una varianza de un $43.792 \%$ (ver tabla 3 ) las condiciones para la interpretación y posteriores análisis son idóneas. Asimismo, aplicada la prueba alfa de Cronbach para cada uno de ellos, refleja que todos gozan de garantías que revelan su consistencia.

Tabla 3: Porcentaje de varianza total explicada por cada factor y fiabilidad resultante del

\begin{tabular}{c|c|c|c|c}
$\mathbf{F}$ & \multicolumn{4}{|c}{ Análisis Factorial } \\
Factor & Varianza explicada & de varianza & \% acumulado & Alfa de Cronbahc \\
\hline $\mathbf{1}$ & 5,817 & 5,540 & 5,540 &, 891 \\
\hline $\mathbf{2}$ & 5,677 & 5,407 & 10,947 &, 883 \\
\hline $\mathbf{3}$ & 3,887 & 3,702 & 14,649 &, 823 \\
\hline $\mathbf{4}$ & 3,646 & 3,472 & 18,121 &, 797 \\
\hline $\mathbf{5}$ & 3,484 & 3,318 & 21,439 &, 815 \\
\hline $\mathbf{6}$ & 3,216 & 3,062 & 24,501 &, 797 \\
\hline $\mathbf{7}$ & 3,090 & 2,943 & 27,444 &, 815 \\
\hline $\mathbf{8}$ & 3,084 & 2,937 & 30,382 &, 740 \\
\hline $\mathbf{9}$ & 2,740 & 2,610 & 32,991 &, 738 \\
\hline $\mathbf{1 0}$ & 2,616 & 2,492 & 35,483 &, 699 \\
\hline $\mathbf{1 1}$ & 2,231 & 2,125 & 37,607 &, 685 \\
\hline $\mathbf{1 2}$ & 2,198 & 2,094 & 39,701 &, 595 \\
\hline $\mathbf{1 3}$ & 2,170 & 2,067 & 41,768 &, 571 \\
\hline $\mathbf{1 4}$ & 2,126 & 2,024 & 43,792 &, 559
\end{tabular}


Con todo ello, los factores que han resultado del análisis aquí realizado, junto con los elementos que los componen, así como la varianza explicada por cada uno de ellos y el valor alfa de Cronbach que estima la fiabilidad de los mismos (todos ellos gozan de garantías que revelan su consistencia), se presentan en la tabla 4.

Tabla 4: Factores obtenidos y aportación al modelo

\begin{tabular}{c|l|c|c} 
Factor & Denominación & $\begin{array}{c}\text { \% de la } \\
\text { varianza }\end{array}$ & $\begin{array}{c}\text { Alfa de } \\
\text { Cronbahc }\end{array}$ \\
\hline $\mathbf{1}$ & Satisfacción personal & 5,540 &, 891 \\
\hline $\mathbf{2}$ & Competencias académicas & 5,407 &, 883 \\
\hline $\mathbf{3}$ & Objetivos de la educación superior & 3,702 &, 823 \\
\hline $\mathbf{4}$ & Derechos del alumnado & 3,472 &, 797 \\
\hline $\mathbf{5}$ & Deberes del alumnado & 3,318 &, 815 \\
\hline $\mathbf{6}$ & Criterios de evaluación del rendimiento académico & 3,062 &, 797 \\
\hline $\mathbf{7}$ & Competencias profesionales & 2,943 &, 815 \\
\hline $\boldsymbol{8}$ & Docencia en red & 2,937 &, 740 \\
\hline $\mathbf{9}$ & Valoración del sistema de tutorías & 2,610 &, 738 \\
\hline $\mathbf{1 0}$ & Objetivos del sistema de tutorías & 2,492 &, 699 \\
\hline $\mathbf{1 1}$ & Objetivos de la evaluación del rendimiento académico & 2,125 &, 685 \\
\hline $\mathbf{1 2}$ & Demandas en la acción tutorial & 2,094 &, 595 \\
\hline $\mathbf{1 3}$ & Cumplimiento de la programación docente & 2,067 &, 571 \\
\hline $\mathbf{1 4}$ & Satisfacción con la evaluación del rendimiento académico & 2,024 &, 559
\end{tabular}

En definitiva, las dimensiones que desde la perspectiva del alumnado encuestado en las Universidades de Córdoba y Salamanca incidirán en la concepción de una formación universitaria de calidad y, en definitiva, claves para la inclusión del sistema universitario español en el Espacio Europeo de Educación Superior, son:

- La satisfacción del alumnado (ver tabla 5), principal índice de producto para valorar la calidad de las instituciones universitarias y finalidad perseguida en el proceso de convergencia europea, es considerada por los participantes en este estudio como el principal componente a la hora de llevar a cabo sistemas de evaluación universitaria. Ésta ha de caracterizarse, en primer lugar, por percibir una enseñanza de calidad, donde los materiales utilizados en el aula sean los adecuados a sus necesidades y donde el plan de estudios a cursar cubra las expectativas de la profesión para la que se están formando. 
Tabla 5: Factor 1: La satisfacción personal

\begin{tabular}{|c|c|}
\hline Ítems de cada Factor & Carga Factorial \\
\hline 131. La enseñanza que recibo es de calidad & ,707 \\
\hline 128. Los materiales utilizados en clase son adecuados & 683 \\
\hline $\begin{array}{l}\text { 129. Este plan de estudios cubre las necesidades de la profesión que } \\
\text { ejerceré en el futuro }\end{array}$ & 679 \\
\hline 115. Confío en esta universidad & 630 \\
\hline 127. La mayoría de las clases que recibo son muy interesantes &, 575 \\
\hline $\begin{array}{l}\text { 119. Mis expectativas con respecto a la formación que estoy reci- } \\
\text { biendo se están cumpliendo satisfactoriamente }\end{array}$ &, 571 \\
\hline 124. Las instalaciones se adecuan a mis necesidades & ,561 \\
\hline $\begin{array}{l}\text { 123. Me siento satisfecho con los servicios y actividades extraacadé- } \\
\text { micas ofertados en la universidad }\end{array}$ & ,480 \\
\hline $\begin{array}{l}\text { 126. Los órganos de gobierno y representación solucionan los pro- } \\
\text { blemas adecuadamente }\end{array}$ & ,441 \\
\hline 118. Me siento bien atendido por el profesorado & ,427 \\
\hline 121. La evaluación responde a todos los aspectos de mi formación & 407 \\
\hline Varianza total explicada & 5,540 \\
\hline Alfa de Cronbach & ,891 \\
\hline
\end{tabular}

- La formación basada en competencias académicas (ver tabla 6), es decir, aquellas adquisiciones que el alumno ha de conseguir tras su paso por la institución universitaria, ha de estar caracterizada por una formación integral basada en habilidades como la reflexión y el autoaprendizaje, en la adquisición de estrategias para resolver conflictos y en el establecimiento de las bases para un aprendizaje continuo.

Tabla 6: Factor 2: Competencias académicas

\begin{tabular}{l|c} 
Ítems de cada Factor & Carga Factorial \\
\hline $\begin{array}{l}\text { 50. Mi paso por la universidad se está formando como persona, no } \\
\text { sólo como un profesional }\end{array}$ &, 718 \\
\hline 62. Estoy desarrollándome personalmente para la vida adulta &, 701 \\
\hline 54. Estoy desarrollando la habilidad de reflexionar y aprender &, 667 \\
\hline 51. Estoy adquiriendo estrategias para la resolución de conflictos &, 645 \\
\hline 55. Estoy aprendiendo a trabajar en contextos diversos &, 638 \\
\hline 64. Estoy estableciendo las bases para un aprendizaje continuo &, 621 \\
\hline 53. Estoy desarrollando técnicas de comunicación &, 549 \\
\hline $\begin{array}{l}\text { 63. Comprendo la metodología y procedimientos de investigación } \\
\text { específicos de las disciplinas }\end{array}$ &, 548 \\
\hline $\begin{array}{l}\text { 52. Estoy desarrollando habilidades y competencias profesionales } \\
\text { derivadas de experiencias de primera mano }\end{array}$ &, 500 \\
\hline $\begin{array}{l}\text { 65. Estoy adquiriendo conocimientos complementarios de nuevas } \\
\text { tecnologías }\end{array}$ &, 457 \\
\hline 57. Estoy aprendiendo a trabajar en equipo &, 432 \\
\hline
\end{tabular}




\begin{tabular}{l|c}
\hline $\begin{array}{l}\text { 56. Estoy adquiriendo experiencias derivadas de la aplicación profe- } \\
\text { sional de los conocimientos }\end{array}$ &, 436 \\
\hline Varianza total explicada & $\mathbf{5 , 4 0 7}$ \\
\hline Alfa de Cronbach & $\mathbf{, 8 8 3}$
\end{tabular}

- La Universidad ha de agrupar, entre sus objetivos (ver tabla 7), la cooperación en el desarrollo de todos los pueblos, así como a la mejora de los sistemas educativos. Un aspecto a destacar es que los alumnos y las alumnas tienen claro que la Universidad ha de ser un lugar de estímulo intelectual en todos los ámbitos, que asesore a la sociedad y favorezca su desarrollo, transmita los saberes de forma crítica y a través de la discusión científica. En definitiva, la Universidad ha de ser un instrumento al servicio de la sociedad.

Tabla 7: Factor 3: Objetivos de la Educación superior

\begin{tabular}{l|c} 
Ítems de cada Factor & Carga Factorial \\
\hline $\begin{array}{l}\text { 28. Cooperar en el desarrollo científico, técnico, cultural y social de } \\
\text { todos los pueblos }\end{array}$ &, 710 \\
\hline 25. Contribuir a la mejora del sistema educativo &, 705 \\
\hline 26. Estimular la actividad intelectual en todos los ámbitos &, 700 \\
\hline 27. Asesorar a la sociedad de modo que se satisfagan sus necesidades &, 685 \\
\hline $\begin{array}{l}\text { 23. Transmitir de forma crítica el saber a través de la actividad docen- } \\
\text { te y la discusión científica }\end{array}$ &, 605 \\
\hline 21. Transmitir la cultura y los valores de la sociedad &, 590 \\
\hline $\begin{array}{l}\text { 24. Capacitar a los individuos para desempenar las funciones que } \\
\text { demanda la especialización laboral }\end{array}$ &, 522 \\
\hline Varianza total explicada & $\mathbf{3 , 7 0 2}$ \\
\hline Alfa de Cronbach & $\mathbf{8 2 3}$
\end{tabular}

- Un aspecto a tener en cuenta en la promoción de sistemas de evaluación para garantizar la calidad de la educación es atender de forma efectiva a los derechos del alumnado universitario (ver tabla 8), entre los que cabe señalar la recepción gratuita de los programas de las asignaturas, la valoración objetiva de su rendimiento académico, el conocimiento de la oferta docente, así como la información sobre todo tipo de becas y ayudas al estudio. 
Tabla 8: Factor 4: Derechos del alumnado

\begin{tabular}{l|c} 
Ítems de cada Factor & Carga Factorial \\
\hline 37. Recibir gratuitamente los programas de las asignaturas &, 714 \\
\hline $\begin{array}{l}\text { 36. Recibir una valoración objetiva del rendimiento académico y } \\
\text { conocer los criterios de valoración del mismo }\end{array}$ &, 702 \\
\hline $\begin{array}{l}\text { 35. Conocer con suficiente antelación la oferta docente y las fechas } \\
\text { de evaluación }\end{array}$ &, 694 \\
\hline $\begin{array}{l}\text { 38. Recibir información sobre todo tipo de becas y ayudas al estudio } \\
\text { y formar parte de las comisiones que las otorguen }\end{array}$ &, 642 \\
\hline \begin{tabular}{l} 
34. Ser atendidos mediante un sistema de tutorías \\
\hline $\begin{array}{l}\text { 39. Disponer de instalaciones acedadas para el adecuado desarrollo } \\
\text { de los estudios y actividades culturales y deportivos }\end{array}$
\end{tabular} &, 498 \\
\hline $\begin{array}{l}\text { 42. Recibir una adecuada información sobre derechos como estudian- } \\
\text { te, así como el funcionamiento general de la Universidad }\end{array}$ &, 467 \\
\hline Varianza total explicada & $\mathbf{3 , 4 7 2}$ \\
\hline Alfa de Cronbach & $\mathbf{7 9 7}$
\end{tabular}

- Por su parte, hay que destacar la importancia que el alumnado otorga al cumplimiento de sus obligaciones, a sus deberes como estudiantes (ver tabla 9). Ellos se centran en el respeto a las normas y el patrimonio de la Universidad, la cooperación para el buen funcionamiento de la institución así como para la consecución de sus objetivos.

Tabla 9: Factor 5: Deberes del alumnado

\begin{tabular}{l|c} 
Ítems de cada Factor & Carga Factorial \\
\hline 44. Respetar las normas y el patrimonio de la universidad &, 695 \\
\hline $\begin{array}{l}\text { 47. Cooperar con el resto de la comunidad universitaria en el buen } \\
\text { funcionamiento de la universidad y en la mejora de sus servicios }\end{array}$ &, 674 \\
\hline $\begin{array}{l}\text { 48. Cooperar par la consecución de los objetivos de la institución } \\
\text { 45. Realizar el trabajo propio del universitario }\end{array}$ &, 668 \\
\hline $\begin{array}{l}\text { 46. Asumir las responsabilidades de los puestos para los que son } \\
\text { elegidos }\end{array}$ &, 661 \\
\hline Varianza total explicada &, 658 \\
\hline Alfa de Cronbach & $\mathbf{3 , 3 1 8}$ \\
\hline
\end{tabular}

- Una formación de calidad está íntimamente relacionada con la evaluación del rendimiento académico (ver tabla 10), aspecto del que el alumnado destaca los criterios como, por ejemplo, la evaluación por medio de actividades de grupo, exposiciones orales o trabajos individuales. 
Tabla 10: Factor 6: Criterios de evaluación del rendimiento académico

\begin{tabular}{l|c} 
Ítems de cada Factor & Carga Factorial \\
\hline 98. En la evaluación se incluyen las actividades de grupo &, 822 \\
\hline 99. En la evaluación se incluyen las exposiciones orales &, 793 \\
\hline 97. El profesorado incluye en la evaluación los trabajos individuales &, 711 \\
\hline $\begin{array}{l}\text { 79. El profesorado incluye actividades de grupo en el desarrollo de } \\
\text { las asignaturas }\end{array}$ &, 547 \\
\hline Varianza total explicada & $\mathbf{3 , 0 6 2}$ \\
\hline Alfa de Cronbach & $\mathbf{, 7 9 7}$
\end{tabular}

- El desarrollo de competencias profesionales (ver tabla 11) es un aspecto destacado en las políticas de gestión de calidad derivada del proceso de convergencia europea y hecho patente por los participantes en este estudio. Estas se plantean como actitudes y cualidades personales, conocimientos específicos y las vivencias derivadas de experiencias de primera mano.

Tabla 11: Factor 7: Competencias profesionales

\begin{tabular}{l|c} 
Ítems de cada Factor & Carga Factorial \\
\hline $\begin{array}{l}\text { 60. Estoy adquiriendo normas, actitudes y cualidades personales } \\
\text { específicas para la profesión }\end{array}$ &, 684 \\
\hline $\begin{array}{l}\text { 59. Los conocimientos que estoy recibiendo están ligados con el } \\
\text { perfil profesional de mi titulación }\end{array}$ &, 616 \\
\hline $\begin{array}{l}\text { 61. Estoy adquiriendo el marco conceptual de las materias de la } \\
\text { titulación }\end{array}$ &, 600 \\
\hline $\begin{array}{l}\text { 58. Estoy conociendo en profundidad algunos aspectos de las mate- } \\
\text { rias con vistas a la especialización }\end{array}$ &, 578 \\
\hline $\begin{array}{l}\text { 56. Estoy adquiriendo experiencias derivadas de la aplicación profe- } \\
\text { sional de los conocimientos }\end{array}$ &, 485 \\
\hline $\begin{array}{l}\text { 52. Estoy desarrollando habilidades y competencias profesionales } \\
\text { derivadas de experiencias de primera mano }\end{array}$ &, 477 \\
\hline Varianza total explicada & $\mathbf{2 , 9 4 3}$ \\
\hline Alfa de Cronbach & $\mathbf{8 1 5}$
\end{tabular}

- La metodología docente basada en sistemas virtuales o en red es uno de los parámetros perseguidos en el proceso de convergencia y destacado aquí por el alumnado (ver tabla 12). Es por ello que consideran necesario el empleo de plataformas virtuales para al docencia, la disponibilidad de materiales didácticos en la red y sistemas de enseñanza semipresencial. 
Tabla 12: Factor 8: Docencia en red

\begin{tabular}{l|c} 
Ítems de cada Factor & Carga Factorial \\
\hline 75. El profesorado utiliza plataformas para la docencia en red &, 783 \\
\hline $\begin{array}{l}\text { 74. Los materiales didácticos de las asignaturas están colgados en la } \\
\text { red }\end{array}$ &, 756 \\
\hline 76. El profesorado utiliza la enseñanza semipresencial &, 594 \\
\hline $\begin{array}{l}\text { 73. El profesorado utiliza recursos multimedia para el desarrollo de la } \\
\text { docencia }\end{array}$ &, 483 \\
\hline $\begin{array}{l}\text { 77. El profesorado emplea metodologías que favorecen el aprendizaje } \\
\text { autónomo del alumnado }\end{array}$ &, 426 \\
\hline Varianza total explicada & $\mathbf{2 , 9 3 7}$ \\
\hline Alfa de Cronbach & $\mathbf{, 7 4 0}$
\end{tabular}

- La acción tutorial se convierte, a partir de la Declaración de Bolonia, en uno de los pilares para la consecución de una formación universitaria de calidad (ver tabla 13). En este sentido, el alumnado considera importante realizar una valoración de dichos sistemas, su funcionamiento, el horario y los niveles generales de satisfacción.

Tabla 13: Factor 9: Valoración del sistema de tutorías

\begin{tabular}{l|c} 
Ítems de cada Factor & Carga Factorial \\
\hline $\begin{array}{l}\text { 107. El sistema actual de las tutorías en la Universidad funciona } \\
\text { adecuadamente }\end{array}$ &, 692 \\
\hline 106. El horario destinado a las tutorías suele ser el adecuado &, 686 \\
\hline 130. Estoy satisfecho con el sistema de las tutorías &, 605 \\
\hline 109. El profesorado lleva a cabo sesiones de tutoría individualizadas &, 420 \\
\hline Varianza total explicada & $\mathbf{2 , 6 1 0}$ \\
\hline Alfa de Cronbach & $\mathbf{, 7 3 8}$
\end{tabular}

- Por su parte, los objetivos del sistema de tutorías han de estar centrados en la información y orientación académica, personal y laboral, ya que son una contribución significativa a la formación del alumnado (ver tabla 14). 
Tabla 14: Factor 10: Objetivos del sistema de tutorías

\begin{tabular}{l|c} 
Ítems de cada Factor & Carga Factorial \\
\hline $\begin{array}{l}\text { 112. El objetivo de las tutorías es orientar e informar sobre aspectos } \\
\text { profesionales }\end{array}$ &, 751 \\
\hline $\begin{array}{l}\text { 113. El objetivo de las tutorías es orientar sobre aspectos personales } \\
\text { 111. El objetivo de las tutorías es orientar e informar sobre aspectos }\end{array}$ &, 719 \\
académicos &, 474 \\
\hline $\begin{array}{l}\text { 114. Las tutorías contribuyen de forma significativa a la formación } \\
\text { del alumnado }\end{array}$ &, 452 \\
\hline Varianza total explicada & $\mathbf{2 , 4 9 2}$ \\
\hline Alfa de Cronbach & $\mathbf{6 9 9}$
\end{tabular}

- Asimismo, los objetivos de la evaluación del rendimiento académico han de convertirse, no en sistemas de sanción, sino en medios de expresión de habilidades y destrezas, de construcción de discursos reflexivos y críticos (ver tabla 15).

\section{Tabla 15: Factor 11: Objetivos de la evaluación del rendimiento académico}

\begin{tabular}{l|c} 
Ítems de cada Factor & Carga Factorial \\
\hline $\begin{array}{l}\text { 102. La evaluación es un medio de expresión de habilidades y destre- } \\
\text { zas }\end{array}$ &, 596 \\
\hline $\begin{array}{l}\text { 101. El objetivo de la evaluación es construir un discurso reflexivo y } \\
\text { crítico }\end{array}$ &, 582 \\
\hline Varianza total explicada & $\mathbf{2 , 1 2 5}$ \\
\hline Alfa de Cronbach & $\mathbf{, 6 8 5}$
\end{tabular}

- Otro elemento a tener en cuenta en este sistema de evaluación de la calidad universitaria, dentro del marco de la acción tutorial, son las demandas del alumnado en los período reservados a dichas actividades (ver tabla 16). Estas se centran principalmente en aclaración de dudas y consultas sobre las actividades docentes e información sobre la evaluación del rendimiento académico.

\section{Tabla 16: Factor 12: Demandas en la acción tutorial}

\begin{tabular}{l|c} 
Ítems de cada Factor & Carga Factorial \\
\hline $\begin{array}{l}\text { 104. Asisto a las tutorías principalmente para realizar consultas y } \\
\text { aclarar dudas }\end{array}$ &, 772 \\
\hline 103. Asisto regularmente a las tutorías &, 703 \\
\hline 105. Asisto a las tutorías principalmente para revisar la evaluación &, 480 \\
\hline Varianza total explicada & $\mathbf{2 , 0 9 4}$ \\
\hline Alfa de Cronbach & $\mathbf{, 5 9 5}$
\end{tabular}


- Por otra parte, el alumnado es consciente de que una institución, para ser catalogada como excelente, ha de ser aquélla en la que el profesorado cumpla su programación docente, tanto práctica como teóricamente (ver tabla 17).

\section{Tabla 17: Factor 13: Cumplimiento de la programación docente}

\begin{tabular}{l|c} 
Ítems de cada Factor & Carga Factorial \\
\hline $\begin{array}{l}\text { 68. La programación práctica que incluye el programa se cumple, al } \\
\text { menos, en el } 80 \%\end{array}$ &, 623 \\
\hline $\begin{array}{l}\text { 67. Los programas teóricos se cumplen, al menos, en un } 80 \% \text { de los } \\
\text { programado }\end{array}$ &, 596 \\
\hline $\begin{array}{l}\text { 94. En los exámenes por escrito el profesorado emplea preguntas } \\
\text { cortas }\end{array}$ &, 440 \\
\hline Varianza total explicada & $\mathbf{2 . 0 6 7}$ \\
\hline Alfa de Cronbach & $\mathbf{, 5 7 1}$
\end{tabular}

- Por ultimo, destacar que el alumnado ha señalado como criterio de calidad su satisfacción son el sistema de evaluación de su rendimiento académico, en tanto las calificaciones conseguidas concuerden con los esfuerzos realizados, los exámenes sean coherentes con la programación docente y los períodos de examen sean adecuados (ver tabla 18).

Tabla 18: Factor 14: Satisfacción con el sistema de evaluación del rendimiento académico

\begin{tabular}{l|c} 
Ítems de cada Factor & Carga Factorial \\
\hline $\begin{array}{l}\text { 122. Las notas que he recibido concuerdan con los esfuerzos realiza- } \\
\text { dos }\end{array}$ &, 706 \\
\hline $\begin{array}{l}\text { 90. Los exámenes son coherentes con los programación de las asigna- } \\
\text { turas }\end{array}$ &, 608 \\
\hline 89. Los períodos reservados a los exámenes son adecuados &, 550 \\
\hline Varianza total explicada & $\mathbf{2 , 0 2 4}$ \\
\hline Alfa de Cronbach & $\mathbf{, 5 5 9}$
\end{tabular}




\section{Discusión}

El Espacio Europeo de Enseñanza Superior, tal y como declara el MECD (2003), es un reto muy positivo para todos. Los estudios tendrán mayor transparencia y comparabilidad con beneficios para toda la sociedad y aportará a los estudiantes una formación basada en sus posibilidades. Existe el convencimiento de que los estudiantes europeos deben ser dotados de una bagaje cultural e intelectual que suponga para ellos la construcción de una vida personal y social significativa y satisfactoria. Es por ello que la calidad, tal y como se señala en el proyecto Tuning (González y Wagenaar, 2003: 59), supone el grado de éxito logrado por la educación superior para generar ambientes apropiados para la producción y transferencia de conocimientos y competencias genéricas y específicas a cada disciplina y a nuevos tipos de aprendizaje.

Las diferentes pruebas llevadas a cabo sobre los datos ofrecidos por el grupo de alumnos y alumnas encuestados han evidenciado que la calidad se define, en primer lugar, por la satisfacción del alumnado, en tanto se trata del principal beneficiario de la formación universitaria y es el usuario principal de sus diferentes servicios. Estos resultados vienen a refrendar, de alguna manera, la lógica de los modelos de evaluación institucional implementados en España hasta ahora aunque, en este estudio, son las opiniones de los estudiantes las que han servido de base para valorar la calidad de la Universidad dentro del marco generado por el proceso de convergencia europea. A continuación, este análisis ha revelado otra serie de dimensiones para la toma de decisiones como son, por una parte, la formación académica basada en la adquisición de habilidades como la reflexión y el autoaprendizaje, la adquisición de estrategias para resolver conflictos y el establecimiento de las bases para el aprendizaje continuo. Por otro lado, estiman necesario el desarrollo de competencias ligadas a la consecución del empleo para el que se están formando. Asimismo, una institución de calidad ha de contar con un profesorado preparado y consciente de su función, un conjunto de metodologías plural que combine presencialidad y virtualidad, unos sistemas de evaluación del rendimiento coherentes y objetivos y un sistema de orientación y acción tutorial que responda a las demandas del alumnado. Igualmente, una universidad de calidad ha de primar, entre sus objetivos, la cooperación y el desarrollo de todos los pueblos, donde se estimule al alumnado crítica, intelectual y culturalmente. 
En definitiva, este estudio ha servido de base, entre otras estrategias, para la generación de un modelo con los indicadores más significativos que, desde el punto de vista de los estudiantes, definen una institución de calidad. Su naturaleza es eminentemente cualitativa, donde la fuente de información ha de ser el alumno universitario y donde el instrumento adecuado para recoger la información puede ser un protocolo estandarizado de evaluación compuesto por ítems de valoración escalar. Este modelo está presenta en la página web: http://www.mec.es/univ/proyectos2005/EA2005-0152.pdf 


\section{Referencias}

Álvarez, V. (2000). Propuestas del profesorado bien evaluado para potenciar el aprendizaje de los estudiantes. Sevilla: Instituto Universitario de Ciencias de la Educación de la Universidad de Sevilla.

Álvarez, M. (1998). El liderazgo de la calidad total. Madrid: Escuela Española.

Apodaca, P.M. y Grao, J. (1996). Autoevaluación, planificación estratégica y calidad total. En F.J. Tejedor y J.L. Rodríguez (Eds.). Evaluación educativa II: Evaluación Institucional. Fundamentos teóricos y aplicaciones prácticas. Salamanca: Instituto Universitario de Ciencias de la Educación de la Universidad de Salamanca.

Chadwick, C.B. y Thorne de Trelles, C. (1998). Calidad de la educación. Ponderación de variables. Internacional Society for Performance Improvement. http://www.pigncispi.com/articles/quality/chadwick-educacion.htm\#op (consultado el 18 de febrero de 2005)

Comunicado de Bergen (2005). The European Higher Education Area. Achieving the Golas. Begern, 19-20 de mayo de 2005. http://www.mec.es/univ/html/EEES_2003/050520_Bergen_Communique.pdf (16 de septiembre de 2005).

Comunicado de Praga (2001). Toward the European Higher Education Area. Communiqué of the meeting of European Ministers in charge of Higher Education. Praga, 19 de mayo de 2001. http://www.univ.mecd.es/univ/html/informes/bolonia/Comunicado_Praga_2001.pdf (19 de noviembre de 2005).

Conferencia de Berlín (2003). Realising the European Higher Education Area. Communiqué of the Conference of Ministers responsible of Higher Education. Berlin, 19 de septiembre de 2003. http://www.usal.es/webusal/Novedades/noticias/bolonia/berlin.pdf (19 de noviembre de 2005).

Convención de Salamanca (2001). Perfilando el Espacio Europeo de la Enseñanza Superior. Mensaje de la Convención de Instituciones Europeas de Educación Superior. Salamanca, 29 y 30 de marzo de 2001.

http://www.usal.es/webusal/Novedades/noticias/bolonia/convencion sal.pdf (19 de noviembre de 2005).

Declaración de Bolonia (1999). The European Higher Education Area. Bologna Declaration. Joint declaration of the European Ministers of Education. Bolonia, 19 de junio de 
1999. http://www.univ.mecd.es/univ/html/informes/bolonia/Declaracion_Bolonia.pdf (19 de noviembre de 2005).

García, E.; Gil, J. y Rodríguez, G. (2000). Análisis Factorial. Madrid: La Muralla / Salamanca: Hespérides.

Gento, S. (1996). Instituciones educativas para la Calidad Total. Madrid: La Muralla.

González, I. (2004). Calidad en la Universidad: evaluación e indicadores. Salamanca: Universidad de Salamanca.

González, J. y Wagenaar, J. (eds.) (2003). Tuning Educational Structures in Europe. Informe Final Fase Uno. Bilbao: Universidad de Deusto.

Hernández, F. (1997). La evaluación de los alumnos en el contexto de la calidad de las universidades. Revista de Investigación Educativa, 14 (2), 25-50.

Martín, M. (2001). La calidad en los centros educativos: el Modelo Europeo de la Calidad y los Planes de Mejora de la Calidad como propuesta de acción. En M. Martín Bris (Coord.). La calidad de la educación en un mundo globalizado: intercambio de experiencias y perspectivas (pp. 17-43). Madrid: Universidad de Alcalá.

MECD (2003). La integración del sistema universitario español en el Espacio Europeo de enseñanza Superior. Madrid: MECD.

Renau, E. (2004). Las claves del éxito de la evaluación institucional. Cuadernos para la Educación Superior, http://www.depcuadernos.net/interface/asp/web/article fitxa.asp?ArticleID=113 (14 de diciembre de 2005)

Rodríguez, S. (1991). Calidad universitaria: un enfoque institucional y multidimensional. En M. De Miguel y S. Rodríguez. La evaluación de las instituciones universitarias (pp. 39-72). Madrid: Consejo de Universidades.

Rodríguez, T. (1998). Finalidades de un centro educativo. Aula Abierta, 71, 2-12.

Ruiz, J. (1996). La investigación educativa. Factor de calidad de la educación. En M. Pérez y J. Ruiz (Coords.). Factores que favorecen la calidad educativa (pp. 43-67). Jaén: Universidad de Jaén. 